\title{
Synthetic Rashba spin-orbit system using a silicon metal-oxide semiconductor
}

\section{Sooboem Lee}

Kyoto University

Hayato Koike

TDK Corporation

\section{Minori Goto}

Osaka University

\section{Shinji Miwa}

University of Tokyo https://orcid.org/0000-0001-9131-6753

Yoshishige Suzuki

Osaka University

Naoto Yamashita

Kyoto University

Ryo Ohshima

Kyoto University

\section{Ei Shigematsu}

Kyoto University

\section{Yuichiro Ando}

Kyoto University

Masashi Shiraishi ( $D$ mshiraishi@kuee.kyoto-u.ac.jp )

Kyoto University

\section{Article}

Keywords: spin-orbit interaction (SOI), SOI systems

Posted Date: December 31st, 2020

DOl: https://doi.org/10.21203/rs.3.rs-129641/v1

License: (1) This work is licensed under a Creative Commons Attribution 4.0 International License.

Read Full License 
Version of Record: A version of this preprint was published at Nature Materials on June 3rd, 2021. See the published version at https://doi.org/10.1038/s41563-021-01026-y. 


\title{
Synthetic Rashba spin-orbit system using a silicon metal-oxide semiconductor
}

\author{
Soobeom Lee ${ }^{1}$, Hayato Koike ${ }^{2}$, Minori Goto ${ }^{3}$, Shinji Miwa ${ }^{3, \$}$, Yoshishige Suzuki ${ }^{3}$, \\ Naoto Yamashita $^{1}$, Ryo Ohshima $^{1}$, Ei Shigematsu ${ }^{1}$, \\ Yuichiro Ando ${ }^{1}$ and Masashi Shiraishi ${ }^{1, \#}$
}

1. Department of Electronic Science and Engineering, Kyoto University, Kyoto, Kyoto 6158510, Japan.

2. Advanced Products Development Center, TDK Corporation, Ichikawa, Chiba 272-8558, Japan

3. Graduate School of Engineering Science, Osaka University, Toyonaka, Osaka 560-8531, Japan

\# Corresponding author: Masashi Shiraishi (shiraishi.masashi.4w@kyoto-u.ac.jp)

\$ Present address: Institute for Solid State Physics, The University of Tokyo, Kashiwa, Chiba 277-8581, Japan. 
The spin-orbit interaction (SOI), as a manifestation of a relativistic effect in solids, has been attracting great attention in condensed matter physics because of its abundant nature for manipulating and/or converting a spin degree of freedom. Compound materials with bulk inversion symmetry breaking (GaAs, BiTeI, $\mathrm{NbSe}_{2} \ldots$ ), heterostructures consisting of the aforementioned materials (InGaAs/InAlAs, Bi/Ag...) and single heavy elements (Pt, W, Ta, Bi...) are pivotal material systems for generating strong Dresselhaus- and Rashba-type SOI and for shedding light on a wide variety of modern physics such as two-dimensional materials science, superconductivity, magnetoelectrics, spintronics etc. Consequently, however, light elements and materials with bulk inversion symmetry have been outside the scope of the quest for SOI in solids. In this work, we show that a Si metal-oxidesemiconductor (MOS) is a new material stage possessing Rashba-type SOI, although Si is a light element and has lattice inversion symmetry resulting in inherently negligible SOI in bulk form. A strong gate electric field is applied to the Si MOS, and unexpectedly, we observe spin precession of propagating spins in the Si through the formation of an emergent effective magnetic field due to the SOI in the Si MOS. Furthermore, the Rashba parameter $\alpha$ in the system increases linearly up to $9.8 \times 10^{-16} \mathrm{eV} \cdot \mathrm{m}$ for a gate electric field of $0.5 \mathrm{~V} / \mathrm{nm}$, i.e., it is gate tuneable, and the spin splitting $\Delta_{0}(=0.6 \mu \mathrm{eV})$ is equivalent to that in strained GaAs. This finding is a successful establishment of a synthetic Rashba system using a ubiquitous light element with bulk inversion symmetry and pioneers a new family of SOI systems.

The SOI gives rise to a wide variety of fruitful condensed matter physics, and it has been playing a central role in a wide variety of physical phenomena such as spin manipulation without magnetic fields ${ }^{1,2}$, ordinal and inverse spin Hall effects enabling spin conversion ${ }^{3-7}$, the spingalvanic effect and its inverse effect ${ }^{8,9}$, giant spin splitting at an interface and in bulk ${ }^{10,11}$ and Ising Cooper-pairing superconductivity ${ }^{12}$. Breaking the bulk inversion symmetry and/or 
structural inversion symmetry is one of the pivotal approaches to generate SOI. For example, bulk inversion symmetry breaking in strained GaAs allows coherent spin manipulation without an external magnetic field ${ }^{1}$, and structural inversion symmetry breaking induces giant spin splitting at the $\mathrm{Bi} / \mathrm{Ag}$ interface ${ }^{10}$ and in the BiTeI bulk ${ }^{11}$. The other significant material with strong SOI is a single heavy element, where the SOI magnitude is roughly proportional to the fourth power of the atomic number ${ }^{13-15}$. Because of this background, materials with high inversion symmetry and light elements have been outside the scope of SOI physics. Single-layer graphene with a transition metal dichalcogenide (van der Waals heterostructure) ${ }^{16-19}$, bilayer graphene ${ }^{20,21}$ and a subsurface state of $\mathrm{p}-\mathrm{Ge}(111)^{22,23}$ are limited examples, and it is noteworthy that the magnitude of the SOI is not tuneable and/or a specific system is necessary for generating SOI in them. Hence, it would be a great surprise and quite significant to realize a novel, unprecedented and tuneable synthetic SOI system using a light element with high inversion symmetry because such a success would dramatically expand the horizons of SOI physics.

Si has been believed to be an unsuitable material for SOI generation because of its lightness and bulk inversion symmetry, and Si has been attracting attention mainly because of its good spin coherence. However, veiled physical properties can appear when a strong gate electric field is applied. Indeed, strong electric field application via solid and ionic gating materials allowed pioneering novel physics in condensed matter, such as spin manipulation in III-V compound semiconductor heterostructure ${ }^{1}$, superconductivity in oxide insulators ${ }^{24}$, strong modulation of the Curie temperature in nanometre-thick $\mathrm{Co}^{25}$ and gate-tuneable SOI resulting in the tuneable inverse spin Hall effect in nanometre-thick $\mathrm{Pt}^{26}$. Thus, whilst a gate electric field merely plays a role in modulating the conductivity of $\mathrm{Si}$ in conventional Si-based electronic devices, the spin degree of freedom can be inherently modulated by a sufficiently strong gate electric field even in $\mathrm{Si}$, creating a synthetic Rashba spin-orbit system. To test this concept, a thin Si metal-oxide- 
semiconductor (MOS) spin channel with a gate insulator can be a model system because the spin propagation physics is well understood in Si MOS.

When a Rashba field, i.e., emergent effective magnetic field, is successfully generated in a Si MOS channel, spin lifetime anisotropy arises between the lifetime of parallel and perpendicular spins to a channel plane. Thus, to identify whether the synthetic Rashba SOI is generated, the spin lifetime anisotropy as a function of the gate voltage amplitude, $V_{\mathrm{g}}$, and angle of an external magnetic field, $B_{\text {ex }}$, should be examined. Unless a gate electric field is applied in a Si spin channel, $B_{\text {ex }}$ is the sole source of spin precession. Meanwhile, application of a gate electric field gives rise to an emergent effective magnetic field perpendicular to the $k$-vector of the propagating spins as $\gamma \hbar B_{\text {eff }}=2 \alpha(k \times z)$, where $\gamma$ is the gyromagnetic ratio, $\hbar$ is the Dirac constant, $B_{\text {eff }}$ is the emergent effective magnetic field, and $z$ is the unit vector along the direction of the gate electric field. Since $B_{\text {eff }}$ provides an additional contribution to spin precession, the spin lifetimes parallel $\left(\tau_{\mathrm{s}}^{\mathrm{P}}\right)$ and perpendicular $\left(\tau_{\mathrm{s}}^{\perp}\right)$ to the spin channel plane exhibit anisotropy. The most appropriate approach to substantiate the anisotropy is the method established for testing the spin lifetime anisotropy in single-layer graphene ${ }^{27}$ and applied to bilayer graphene ${ }^{20,21}$. Raes et al. established the approach, wherein they applied out-of-plane (oblique) magnetic fields and implemented spin precession measurements under oblique magnetic fields to generate an out-of-plane spin population. The superiority of this method is that it provides reliable results for both low- and high-carrier densities and thus can be employed for gate tuneable systems such as Si MOS.

Figure 1(a) shows the structure of a synthetic Rashba device consisting of a $100 \mathrm{~nm}$-thick n-type Si channel (the carrier concentration, $n$, was $5 \times 10^{16} \mathrm{~cm}^{-3}$, i.e., the $\mathrm{Si}$ was non-degenerate) on a $200 \mathrm{~nm}$-thick $\mathrm{SiO}_{2}$ gate insulator ( $\mathrm{Si} \mathrm{MOS}$ ) and the setup for measurement of the non-local four-terminal magnetoresistance (NL4T-MR) and spin lifetime anisotropy in the Si MOS under 
the application of $V_{\mathrm{g}}$ (see also Methods). All measurements were implemented at $300 \mathrm{~K} . V_{\mathrm{g}}$ was applied from the backside of the device and was varied from $0 \mathrm{~V}$ to $100 \mathrm{~V}$, i.e., an electric field was applied to the $\mathrm{Si}$ from 0 to $0.5 \mathrm{~V} / \mathrm{nm}$ via $\mathrm{SiO}_{2}$. Modulation of the conductivity, $\sigma_{\mathrm{Si}}$, of the $\mathrm{Si}$ MOS was realized as shown in Fig. 1(b), which indicates that the back-gate voltages were efficiently applied to the Si MOS channel. The NL4T-MR from the Si MOS under $V_{\mathrm{g}}=0 \mathrm{~V}$ with sweeping of the in-plane external magnetic field is shown in Fig. 1(c), and clear resistance hysteresis as a manifestation of successful spin transport in the Si MOS is observed. To clarify the spin lifetime anisotropy, $B_{\mathrm{ex}}$ was applied with changing applied angle $\beta$, as shown in Fig. 1(a), where $\beta$ was varied from $10^{\circ}$ to $90^{\circ}$.

Spin precession signals for various $\beta$ (as representatives, $10^{\circ}, 30^{\circ}, 45^{\circ}, 60^{\circ}$, and $90^{\circ}$ ) as a function of $V_{\mathrm{g}}$ (as representatives, $0,10,60$, and $100 \mathrm{~V}$ ) are depicted in Figs. 2(a)-2(d). The signals were nicely reproduced by a conventional one-dimensional fitting function ${ }^{28,29}$, where the spin lifetime at $\beta=90^{\circ}$, i.e., $\tau_{\mathrm{s}}^{\mathrm{P}}$, under $V_{\mathrm{g}}=0 \mathrm{~V}$ was estimated to be $2.5 \pm 0.1 \mathrm{~ns}$, and the precessional motion of the spins dephases at $B_{\mathrm{ex}} \sim 40 \mathrm{mT}$ (see also Supplemental Information No. 1). For $B_{\mathrm{ex}}$ $>40 \mathrm{mT}$, the spin voltage, $V_{\mathrm{NL} 4 \mathrm{~T}}$, is determined by the remanent non-precessional spin component that lies along the magnetic field direction. The angular dependence of normalized $V_{\mathrm{NL} 4 \mathrm{~T}}$ is described as

$$
\frac{V_{\mathrm{NL} 4 \mathrm{~T}}(\beta)}{V_{\mathrm{NL} 4 \mathrm{~T}}\left(0^{\circ}\right)}=\cos ^{2} \beta \sqrt{\left(\cos ^{2} \beta+\zeta^{-1} \sin ^{2} \beta\right)^{-1}} \exp \left(-\sqrt{\frac{L^{2}}{D \tau_{\mathrm{s}}^{\mathrm{P}}}}\left(\sqrt{\cos ^{2} \beta+\zeta^{-1} \sin ^{2} \beta}-1\right)\right),
$$

where $L$ is the centre-to-centre distance between two ferromagnetic contacts, $D$ is the diffusion constant, and $\zeta$ is the spin lifetime anisotropy ratio, $\zeta=\tau_{\mathrm{s}}^{\perp} / \tau_{\mathrm{s}}^{\mathrm{P}}{ }^{26}$. The ratio $\zeta$ is a fingerprint of the anisotropy, where $\zeta<1$ is expected when spin-orbit fields inducing spin relaxation are preferential in the $\mathrm{Si}$ MOS plane, and $\zeta=1$ is observed for an isotropic spin lifetime. Thus, $\zeta$ is quantitatively characterized by plotting the normalized $V_{\mathrm{NL} 4 \mathrm{~T}}$ at $\beta=0^{\circ}$ and $\beta \neq 0^{\circ}$. Figures 2(e)- 
2(h) show the normalized $V_{\mathrm{NL} 4 \mathrm{~T}}$ as a function of $\cos ^{2} \beta$, where the magnitudes of $\zeta$ estimated by using Eq. (1) are also shown. $\zeta$ changes with $V_{\mathrm{g}}$ and is quite close to 1 when $V_{\mathrm{g}}=10 \mathrm{~V}$. The complete dataset of $\zeta$ as a function of $V_{\mathrm{g}}$ is shown in Fig. 3, and a prominent $V_{\mathrm{g}}$ dependence of $\zeta$ is seen. Indeed, $\zeta$ monotonically and prominently decreases when $V_{\mathrm{g}}>10 \mathrm{~V}$, which is unlike SLG ${ }^{26}$. As mentioned above, $\zeta$ is a fingerprint of the spin-orbit fields in $\mathrm{Si}$, and the observed $V_{\mathrm{g}}$ dependence of $\zeta$ unequivocally shows that a spin-orbit field is generated in the Si MOS and that spin lifetime anisotropy arises from emergent effective magnetic fields that are tuneable by $V_{\mathrm{g}}$. Notably, the experimental result counters the conventional understanding that Si has negligibly small SOI, and we have successfully demonstrated not only generation but also manipulation of SOI in the Si MOS by gating. As mentioned above, the Rashba field generates an emergent effective magnetic field. In our setup, the gate electric field is applied perpendicular to the $\mathrm{Si}$ plane (along the $z$-direction, as shown in Fig. 1(a)), and thus, the effective magnetic field is generated in-plane along the $(-y)$-direction because the motion of the propagating spins is along the $x$-direction (see Fig. 4(a)). The in-plane emergent effective magnetic field further helps spin relaxation of the propagating spins, which enhances the spin lifetime anisotropy. Surprisingly, $\zeta$ is quite close to 1 not at $V_{\mathrm{g}}=0 \mathrm{~V}$ but at $V_{\mathrm{g}}=10 \mathrm{~V}$, i.e., the spin lifetime is isotropic at $V_{\mathrm{g}}=10 \mathrm{~V}$. This suggests that a built-in and non-negligible electric field, whose direction is opposite to that of the positive external gate electric field, is generated in the Si spin channel. Thus, the SOI in the Si MOS is ascribable to the thin $(100 \mathrm{~nm})$ channel and/or the $\mathrm{Si} / \mathrm{SiO}_{2}$ interface.

How spins precess under magnetic fields is described by the following equation in the simplest model ${ }^{30}$ :

$$
\omega=\frac{\Delta \theta}{t}=\gamma|B|=\frac{2 \alpha k_{x}}{\hbar},
$$

where $\omega$ is the angular frequency of spin precession, $\Delta \theta$ is the spin precession angle, $t$ is the dwell time of the spins propagating between two electrodes, $\gamma$ is the gyromagnetic ratio, $B$ is the 
magnetic field that spins sense, $k_{x}\left(=\left(3 \pi^{2} n\right)^{\frac{1}{3}}\right)$ is the wavenumber of the propagating spins diffusing along the $x$-direction and $\alpha$ is the Rashba parameter (see also Fig. 4(a)). Thus, the Rashba field strength that determines the strength of the emergent effective magnetic field is quantitatively described by $\alpha$. The upper and lower figures in Fig. 4(b) show enlarged views of the spin precession signals at $\beta=0^{\circ}$ for $V_{\mathrm{g}}=+10 \mathrm{~V}(\zeta=0.99$, isotropic spin lifetime $)$ and $100 \mathrm{~V}$ $(\zeta=0.75$, anisotropic spin lifetime), respectively, and the dashed lines show the external magnetic field, where the averaged spin precession angle is $\pi$ and $-\pi$. We postulate that the Rashba SOI at $V_{\mathrm{g}}=10 \mathrm{~V}$ is negligible and no emergent effective field is generated in the Si MOS, because the external magnetic field for $\pi$-rotation is the smallest when $V_{\mathrm{g}}=10 \mathrm{~V}$ (see also the following discussion). Here, a $B_{\text {ex }}$ of $6.6 \mathrm{mT}$ is necessary for $\pi$-rotation at $V_{\mathrm{g}}=10 \mathrm{~V}$. Nevertheless, a greater $B_{\text {ex }}$ of $10.6 \mathrm{mT}$ is needed for $\pi$-rotation at $V_{\mathrm{g}}=100 \mathrm{~V}$. The enhancement of the external magnetic field, $B_{\text {ex }}$, needed for $\pi$-rotation of the spins, helps in understanding the underlying physics. Since we exerted a non-local four-terminal scheme for spin transport, only spin diffusion occurs. Since $D$ is independent of $V_{\mathrm{g}}$ (see Supplemental Information No. 2), the Fermi velocity and momentum relaxation time are unchanged with $V_{\mathrm{g}}$, resulting in unchanged mobility and dwell time $t$ for $\pi$ rotation of the spins in the Si MOS even when $V_{\mathrm{g}}$ increases up to $100 \mathrm{~V}$. At $V_{\mathrm{g}}=10 \mathrm{~V}$, the $B$ that spins sense can be regarded as merely $B_{\text {ex }}$ because no $B_{\text {eff }}$ arises, and the spin precession angle is determined to be $\Delta \theta=\gamma\left|B_{\mathrm{ex}}\right| t$. Meanwhile, at $V_{\mathrm{g}}=100 \mathrm{~V}$, the $B$ that spins sense is the coupled magnetic field of $B_{\mathrm{ex}}$ and $B_{\mathrm{eff}}$, i.e., $\sqrt{B_{\mathrm{ex}}{ }^{2}+B_{\mathrm{eff}}^{2}}$. Furthermore, the spin precession angle in the anisotropy measurement setup is determined by the difference between $\sqrt{B_{\mathrm{ex}}{ }^{2}+B_{\mathrm{eff}}{ }^{2}}$ and $B_{\mathrm{eff}}$ because the averaged spin precession angle in the measurement setup is determined by the difference in the magnetic fields with and without the external magnetic field, $B_{\text {ex. }}$ Consequently, the averaged spin precession angle at $V_{\mathrm{g}}=100 \mathrm{~V}$ is described as $\Delta \theta=\gamma\left|\left(\sqrt{B_{\mathrm{ex}}{ }^{2}+B_{\mathrm{eff}}{ }^{2}}-B_{\mathrm{eff}}\right)\right| t$. 
Given that the dwell time for $\pi$-rotation is unchanged at $V_{\mathrm{g}}=10 \mathrm{~V}$ and $100 \mathrm{~V}$ and the $B_{\text {ex }}$ for $\pi$ rotation at $V_{\mathrm{g}}=10 \mathrm{~V}$ and $100 \mathrm{~V}$ is $6.6 \mathrm{mT}$ and $10.6 \mathrm{mT}$, respectively, the $B_{\text {eff }}$ at $V_{\mathrm{g}}=100 \mathrm{~V}$ (the electric field of $0.5 \mathrm{~V} / \mathrm{nm}$ ) is estimated to be $5.3 \mathrm{mT}$, resulting in a Rashba parameter, $\alpha$, of $9.8 \times$ $10^{-16} \mathrm{eV} \cdot \mathrm{m} . \alpha$ is continuously tuned as a function of $V_{\mathrm{g}}$ as shown in Fig. 4(d), which reflects the compelling result of a tuneable Rashba SOI in the Si MOS. The spin splitting, $\Delta_{0}\left(=g \mu_{\mathrm{B}} B_{\text {eff, }}\right.$ where $g$ is the g-factor, $\mu_{\mathrm{B}}$ is the Bohr magneton, and $B_{\text {eff }}$ is the emergent effective magnetic field) at $V_{\mathrm{g}}$ $=100 \mathrm{~V}$ for the Si MOS is calculated to be $0.6 \mu \mathrm{eV}$, which is much smaller than that in III-V heterostructures ${ }^{1}$ but is comparable to that in strained GaAs ${ }^{2}$. Although the SOI in GaAs is inherently much greater, the g-factor of $\mathrm{Si}$ (1.9979 in experiment and 1.99875 in theory at room temperature $\left.{ }^{31}\right)$ is approximately five-fold greater than that in $\operatorname{GaAs}\left(0.441^{2}\right)$, which is the reason why comparable spin splitting is realized in the Si MOS despite the small $\alpha$. Notably, twenty-fold enhancement of $\alpha$ can be realized by replacing a thin gate insulator with a greater dielectric constant material, such as $50 \mathrm{~nm} \mathrm{HfO}_{2}{ }^{25}$, and most likely, much greater enhancement may be achieved by using ionic gating, where a strong electric field is applicable ${ }^{26}$. The enhancement would allow larger spin splitting in the Si MOS, as in III-V heterostructures ${ }^{1}$, enabling other spin manipulation functions in Si.

The finding in this study of successful creation of a synthetic Rashba system using a ubiquitous light element counters the conventional understandings in SOI physics, i.e., that the SOI in Si is negligibly small and a synthetic SOI as realized in van der Waals heterostructures cannot be created in Si. Thus, this report pioneers a new approach to creating a novel material system with a sizable SOI. Since the created SOI in Si MOS is gate tuneable and the spin splitting $\Delta_{0}$ is comparable to that in GaAs strained under an application of gate voltages via $200 \mathrm{~nm}$-thick $\mathrm{SiO}_{2}$, further enhancement of $\alpha$ by introducing insulating gate materials with larger dielectric constants can pave the way to novel and more efficient spin manipulation systems using Si. 



\section{Methods}

\section{Device fabrication}

Ferromagnetic contacts $(\mathrm{Fe} / \mathrm{Co} / \mathrm{MgO})$ for spin injection and detection and non-magnetic contacts (Al) as reference electrodes were fabricated on 100 nm-thick n-type $\mathrm{Si}(100)$ ( $\mathrm{Si}$ MOS channel)/200 nm-thick $\mathrm{SiO}_{2} /$ bulk $\mathrm{Si}(100) . \mathrm{MgO}$ was inserted to circumvent the conductance mismatch problem, and the dopant in the n-type $\mathrm{Si}$ was phosphorous $(\mathrm{P})$, which was ion implanted. Prior to the deposition of ferromagnetic metal/tunnel barrier layers, a $20 \mathrm{~nm}$-thick highly doped silicon layer was grown by magnetron sputtering to suppress the formation of a depletion layer. $\mathrm{Al}(3 \mathrm{~nm}) / \mathrm{Fe}(12.4 \mathrm{~nm}) / \mathrm{Co}(0.6 \mathrm{~nm}) / \mathrm{MgO}(0.8 \mathrm{~nm})$ layers were subsequently deposited on the $\mathrm{Si}$ MOS by molecular-beam epitaxy as ferromagnetic electrodes. After deposition of all the layers, the Si MOS channel with two FM contacts was fabricated by electron-beam lithography and argon-ion milling. The sizes of the two FM contacts were $0.2 \times 21 \mu \mathrm{m}^{2}$ and $0.8 \times 21 \mu \mathrm{m}^{2}$. The centre-to-centre distance, $L$, was set to $2 \mu \mathrm{m}$. The top surface of the Si spin channel was etched to remove $20 \mathrm{~nm}$ of the highly doped silicon layer. Finally, two non-magnetic (NM) electrodes were fabricated.

\section{Measurements}

An external magnetic field was applied in the $y-z$ plane, and the tilt angle was defined as $\beta$. Since the ferromagnetic electrodes have shape magnetic anisotropy, in-plane magnetic fields $\left(\beta=0^{\circ}\right.$, along the $y$-axis) were applied for switching the magnetization of the ferromagnetic contacts in the non-local four-terminal magnetoresistance measurements. An oblique magnetic field was applied to estimate the spin lifetime anisotropy in the Si MOS. An external gate voltage $\left(V_{\mathrm{g}}\right)$ was applied from the backside of the $\mathrm{Si}$ substrate via $\mathrm{SiO}_{2}$ to create and tune the Rashba SOI. The amplitude of the injected electric current was set to $0.5 \mathrm{~mA}$ and $1.0 \mathrm{~mA}$ for the non-degenerate and degenerate Si MOS, respectively. 


\section{References}

1. Nitta, J., Akazaki, T., Takayanagi, H. \& Enoki, T. Gate control of spin-orbit interaction in an inverted $\mathrm{In}_{0.53} \mathrm{Ga}_{0.47} \mathrm{As} / \mathrm{In}_{0.52} \mathrm{Al}_{0.48} \mathrm{As}$ heterostructure. Phys. Rev. Lett. 78, 1335-1338 (1997).

2. Kato, Y.K., Myers, R.C., Gossard, A.C. \& Awschalom, D.D. Coherent spin manipulation without magnetic fields in strained semiconductors. Nature 427, 50-53 (2003).

3. Murakami, S., Nagaosa, N. \& Zhang, S-C. Dissipationless quantum spin current at room temperature, Science 301, 1348-1351 (2003).

4. Kato, Y.K., Myers, R.C., Gossard, A.C. \& Awschalom, D.D. Observation of the spin Hall effect in semiconductor. Science 306, 1910-1913 (2004).

5. Valenzuela, S. O. \& Tinkham, M. Direct electronic measurement of the spin Hall effect. Nature 442, 176-179 (2006).

6. Saitoh, E., Ueda, M., Miyajima, H. \& Tatara, G. Conversion of spin current into charge current at room temperature: inverse spin-Hall effect. Appl. Phys. Lett. 88, 182509 (2006).

7. Otani, Y., Shiraishi M., Oiwa, A., Saitoh, E. \& Murakami, S. Spin conversion on the nanoscale. Nature Phys. 13, 829-832 (2017).

8. Ganichev, S.D., Ivchenko, E.L., Bel'kov, V.V., Tarasenko, S.A., Sollinger, M., Weiss, D., Wegscheider, W. \& Prettl, W. Spin-galvanic effect. Nature 417, 153-156 (2002).

9. Rojas-Sanchez, J-C. et al. Spin-to-charge conversion using Rashba coupling at the interface between non-magnetic materials. Nature Commun. 4, 2944 (2013).

10. Ast. C.R., Henk, J., Ernst, A. Moreschini, L., Falub, M.C., Pacile, D., Bruno, P., Kern, K. \& Grioni, M. Giant spin splitting through surface alloying. Phys. Rev. Lett. 98, 186807 (2007).

11. Ishizaka, K. et al. Giant Rashba-type spin splitting in bulk BiTeI. Nature Mater. 10, 521 (2011). 12. de la Barrera, S.C., Sinko, M.R., Gopalan, D.P., Sivadas, N., Seyler, K.L., Watanabe, K., Taniguchi, T., Tsen, A.W., Xu, X., Xiao, D. \& Hunt, B.M. Tuning Ising superconductivity 
with layer and spin-orbit coupling in two-dimensional transition-metal dichalcogenides. Nature Commun. 9, 1427 (2018).

13. Ganguly, A., Kondou, K., Sukegawa, H., Mitani, S., Kasai, S., Niimi, Y., Otani, Y. \& Barman, A. Thickness dependence of spin torque ferromagnetic resonance in $\mathrm{Co}_{75} \mathrm{Fe}_{25} / \mathrm{Pt}$ bilayer films. Appl. Phys. Lett. 104, 072405 (2014).

14. Liu, L., Moriyama, T., Ralph D.C. \& Buhrman, R.A. Spin-torque ferromagnetic resonance induced by the spin Hall effect. Phys. Rev. Lett. 106, 036601 (2011).

15. Pai, C-F., Liu, L., Li, Y., Tseng, H.W., Ralph D.C., \& Buhrman, R.A. Spin transfer torque devices utilizing the giant spin Hall effect of tungsten. Appl. Phys. Lett. 101, 122404 (2012).

16. Ghiasi, T.S., Ingla-Aynes, J., Kaverzin, A.A. \& van Wees, B. Large Proximity-Induced Spin Lifetime Anisotropy in Transition-Metal Dichalcogenide/Graphene Heterostructures. Nano Lett. 17, 7528-7532 (2017).

17. Benitez, L.A., Sierra, J.F., Torres, W.S., Arrighi, A., Bonell, F., Costache, M.V. \& Valenzuela S.O. Strongly anisotropic spin relaxation in graphene-transition metal dichalcogenide heterostructures at room temperature. Nature Phys. 14, 303-308 (2018).

18. Safeer, C.K., Ingla-Aynes, J., Herling, F., Garcia, J.H., Vila, M., Ontoso, N., Calvo, R., Roche, S., Hueso, L.E. \& Casanova, F. Room-Temperature Spin Hall Effect in Graphene/MoS 2 van der Waals Heterostructures. Nano Lett. 19, 1074-1082 (2019).

19. Benitez, L.A., Torres, W.S., Sierra, J.F., Timmermans, M., Garcia, J.H., Roche, S., Costache, M.V. \& Valenzuela, S.O. Tunable room-temperature spin galvanic and spin Hall effects in van der Waals heterostructures. Nature Mater. 19, 170-175 (2020).

20. Xu, J., Zhu, T., Luo, Y.K., Lu, Y-M. \& Kawakami, R.K. Strong and tunable spin-lifetime anisotropy in dual-gated bilayer graphene. Phys. Rev. Lett. 121, 127703 (2018).

21. Leutenantsmeyer, J.C., Ingla-Aynes, J., Fabian, J. \& van Wees, B.J. Observation of spin- 
valley-coupling-induced large spin-lifetime anisotropy in bilayer graphene, Phys. Rev. Lett. 121, $127702(2018)$.

22. Ohtsubo, Y., Hatta, S., Yaji, K., Okuyama, H., Miyamoto, K., Okuda, T., Kimura, A., Namatame, H., Taniguchi, M. \& Aruga, T. Spin-polarized semiconductor surface states localized in subsurface layers. Phys. Rev. B 82, 201307(R) (2010).

23. Guillet, T., Zuchetti, C., Bardediene, Q., Marty, A., Isella, G., Cagnon, L., Verganaud, C. Jaffres, H., Reyren, N., George, J.-M., Fert A. \& Jamet, M. Phys. Rev. Lett. 124, 027201 (2020).

24. Ueno, K., Nakamura, S., Shimotani, H., Ohtomo, A., Kimura, N., Nojima T., Aoki, H., Iwasa, Y. \& Kawasaki, M. Electric-field-induced superconductivity in an insulator. Nature Mater. 7, 855-858 (2008).

25. Chiba, D., Fukami, S., Shimamura, K., Ishiwata, N. \& Ono, T. Electrical control of the ferromagnetic phase transition in cobalt at room temperature. Nature Mater. 10, 853-856 (2011)

26. Dushenko, S., Hokazono, M., Nakamura K., Ando, Y., Shinjo T. \& Shiraishi M. Tunable inverse spin Hall effect in nanometer-thick platinum films by ionic gating. Nature Commun. 9, $3188(2018)$.

27. Raes, B., Scheerder, J.E., Castache, M.V., Bonell, F. Sierra, J.F., Cuppens, J., Van de Vondel, J. \& Valenzuela, S.O. Determination of the spin-lifetime anisotropy in graphene using oblique spin precession. Nature Commun. 7, 11444 (2016).

28. Sasaki, T., Oikawa, T., Suzuki, T., Shiraishi, M., Suzuki, Y. \& Noguchi, K. Temperature dependence of spin diffusion length in silicon by Hanle-type spin precession. Appl. Phys. Lett. 96, $122101(2010)$.

29. O’Brien. L., Spivak, D., Krueger, N., Peterson, T.A., Erickson, M.J., Bolon, B., Geppert, C.C., 
Leighton, C. \& Crowell, P.A. Obervation of and modelling of ferromagnetic contact-induced spin relaxation in Hanle spin precession measurements. Phys. Rev. B 94, 094431 (2016).

30. Datta, S. \& Das, B. Electronic analog of the electro-optic modulator, Appl. Phys. Lett. 56, 665-667 (1990).

31. Konakov, A.A., Ezhevskii, A.A., Soukhorukov, A.V., Guseinov, D.V., Popkov, S.A. \& Burdov, V.A. Lande factor of the conduction electrons in silicon: temperature dependence, J. Phys.: Conf. Ser. 324, 012027 (2011). 


\section{Acknowledgements}

This research is supported in part by the Japan Society for the Promotion of Science (JSPS) Research Fellow Program (No. 18J22869) and a Grant-in-Aid for Scientific Research (S) "Semiconductor Spincurrentronics" (No. 16H06330).

\section{Author contributions}

M. S. and S. L. conceived the experiments. H. K., M. G., S. M., and Y. S. fabricated samples.

S. L. and N. Y. collected data. S. L., R. O., E. S., Y. A., and M. S. analysed the results. S. L. and M. S. wrote the manuscript. All authors discussed the results.

\section{Competing interest}

The authors declare no competing interests.

\section{Additional information}

Supplementary information is available for this paper at XXX.

Correspondence and requests for materials should be addressed to M. S. and S. L. 


\section{Figures and figure captions}

(a)

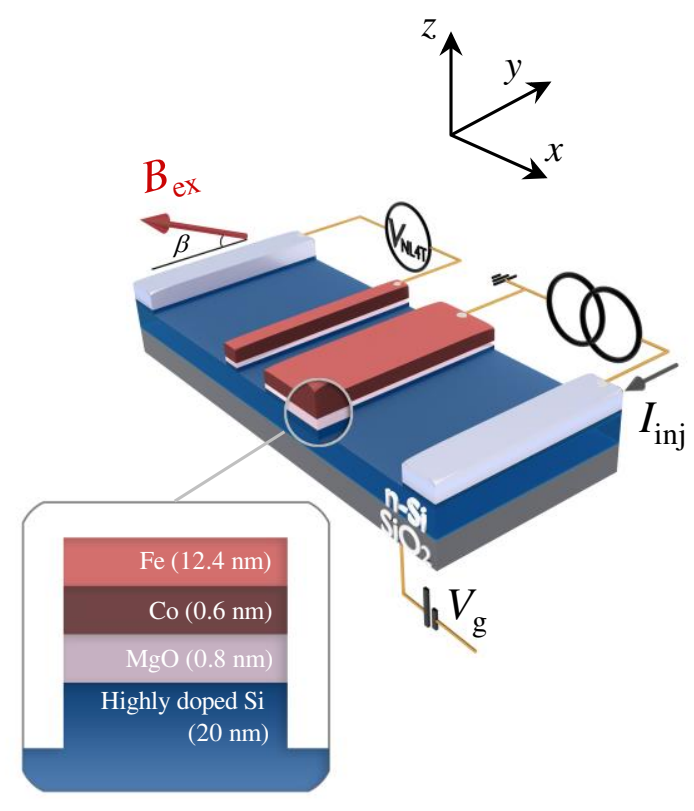

(b)

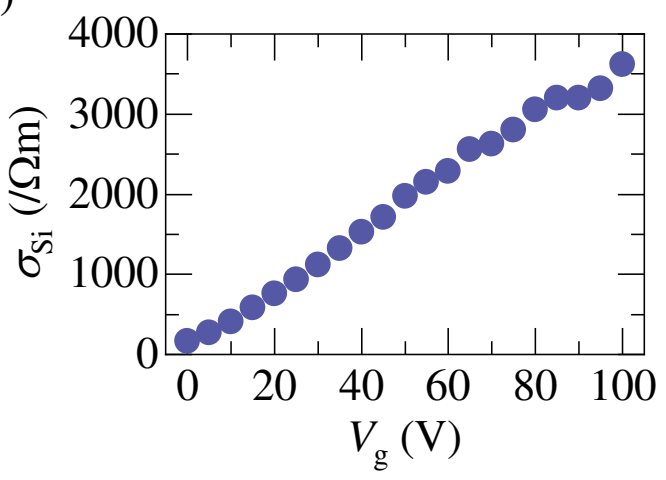

(c)

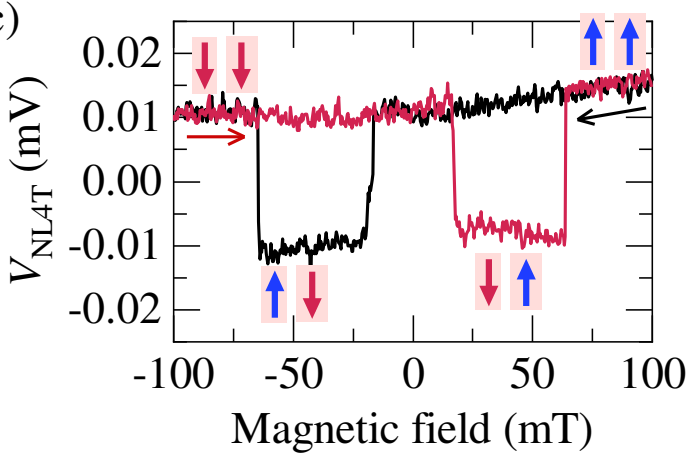

Figure 1 | Device structure and basic charge and spin transport characteristics in a synthetic

Rashba system made of Si MOS. (a) Schematic image of a synthetic Rashba device made of $\mathrm{Si}$ MOS and the measurement setup. The Si MOS channel is non-degenerate and $100 \mathrm{~nm}$ thick. An electric current $\left(I_{\text {inj }}\right)$ is applied between one ferromagnetic contact and one non-magnetic contact, and NL4T voltages $\left(V_{\mathrm{NL} 4 \mathrm{~T}}\right)$ are measured in the separated circuit including a ferromagnetic contact and a non-magnetic contact: non-local alignment. An external magnetic field $\left(B_{\mathrm{ex}}\right)$ is applied in the $y-z$ plane with a tilt angle of $\beta$. (b) Conductivity of the Si MOS $\left(\sigma_{\mathrm{Si}}\right)$ as a function of gate voltage $\left(V_{\mathrm{g}}\right) . \sigma_{\mathrm{Si}}$ was measured using a conventional four-terminal method. (c) Typical non-local magnetoresistance $\left(\Delta V_{\mathrm{NL} 4 \mathrm{~T}}\right)$ as a manifestation of successful spin propagation in the Si MOS. An in-plane $\left(\beta=0^{\circ}\right)$ external magnetic field was swept upwards (red solid line) and downwards (black solid line). 
(a)

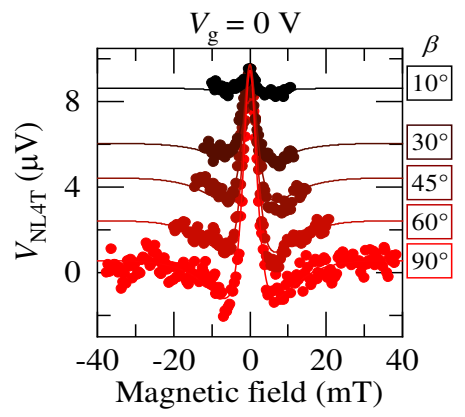

(b)

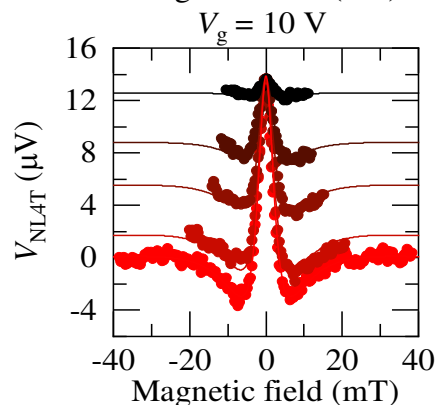

(c)

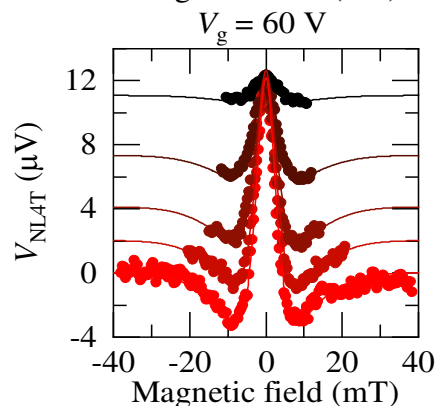

(d)

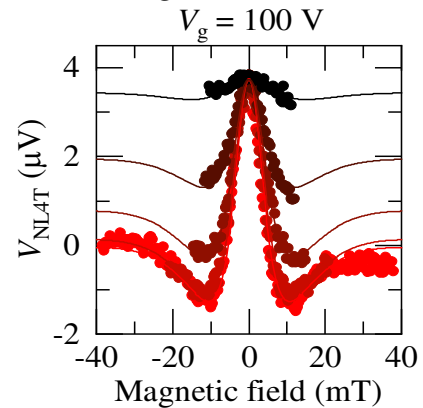

(e)

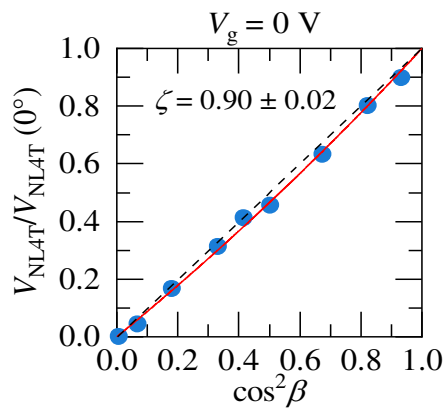

(f)

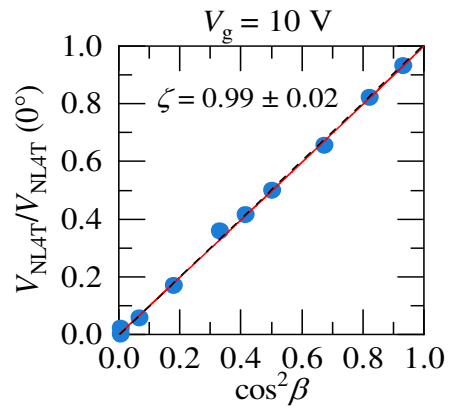

(g)

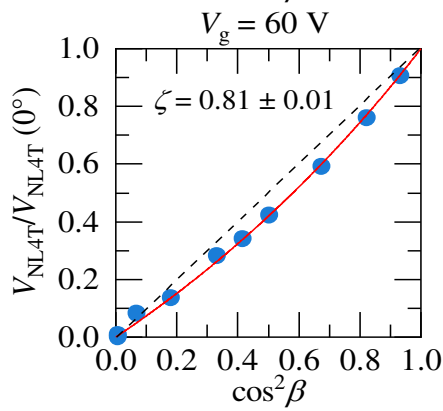

(h)

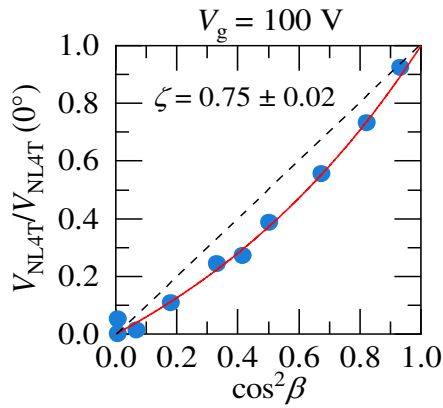

Figure 2 | Gate modulation of spin precession signals and spin lifetime anisotropy. (a)-(d)

Non-local spin signal as a function of tilt angle $\beta$ and gate voltage $V_{\mathrm{g}}$. Solid lines show fitting results obtained using a conventional spin precession equation. (e)-(h) Spin signals under a high magnetic field ( $>40 \mathrm{mT}$ ) at various $\beta$ normalized by those at $\beta=0^{\circ}$ as a function of $\cos ^{2} \beta$. Red solid lines are fitting results obtained by using Eq. (1) in the main text. The anisotropy ratio of the 
spin lifetime ( $\zeta)$ is extracted by fitting and is depicted in the figures. The black dashed lines represent the $\cos ^{2} \beta$ dependence when $\zeta=1$ (isotropic). 


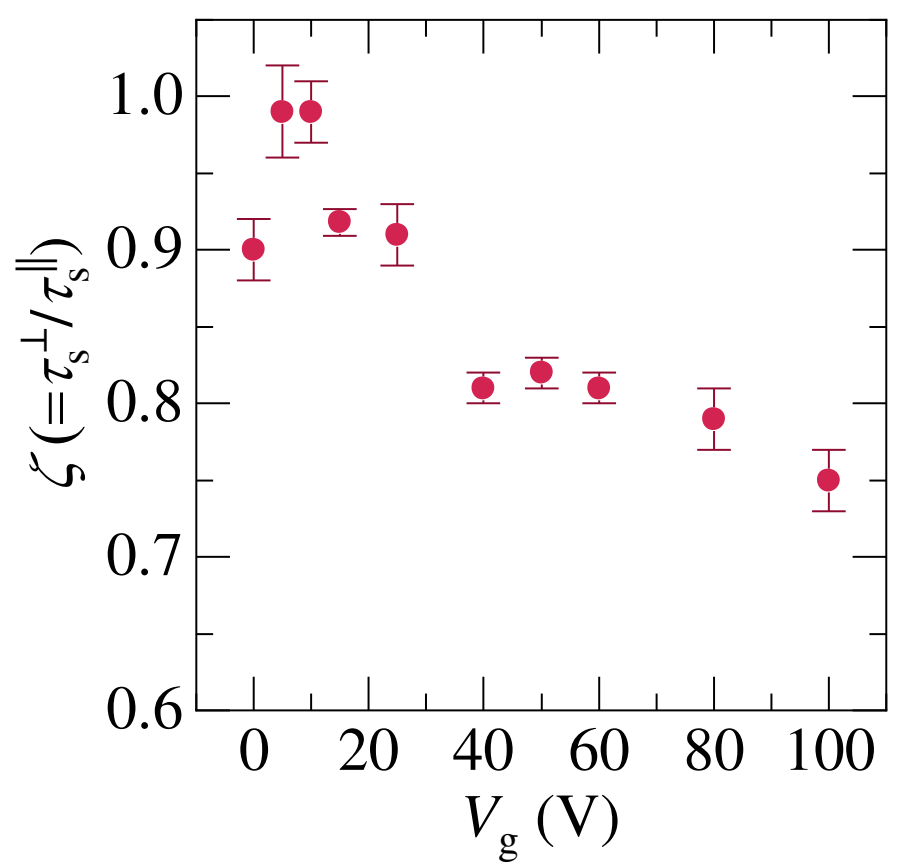

Figure 3 | Gate voltage dependence of the spin lifetime anisotropy. The spin lifetime anisotropy ratio $\zeta$ is dependent on $V_{\mathrm{g}}$, and it is the largest and almost unity at $V_{\mathrm{g}}=10 \mathrm{~V}$, implying an isotropic spin lifetime. Above $V_{\mathrm{g}}=10 \mathrm{~V}, \zeta$ monotonically decreases with increasing $V_{\mathrm{g}}$, which is a manifestation of the generation and enhancement of a synthetic Rashba SOI field, resulting in an emergent effective magnetic field. The error bars are estimated as the standard deviation in the fitting. 
(a)

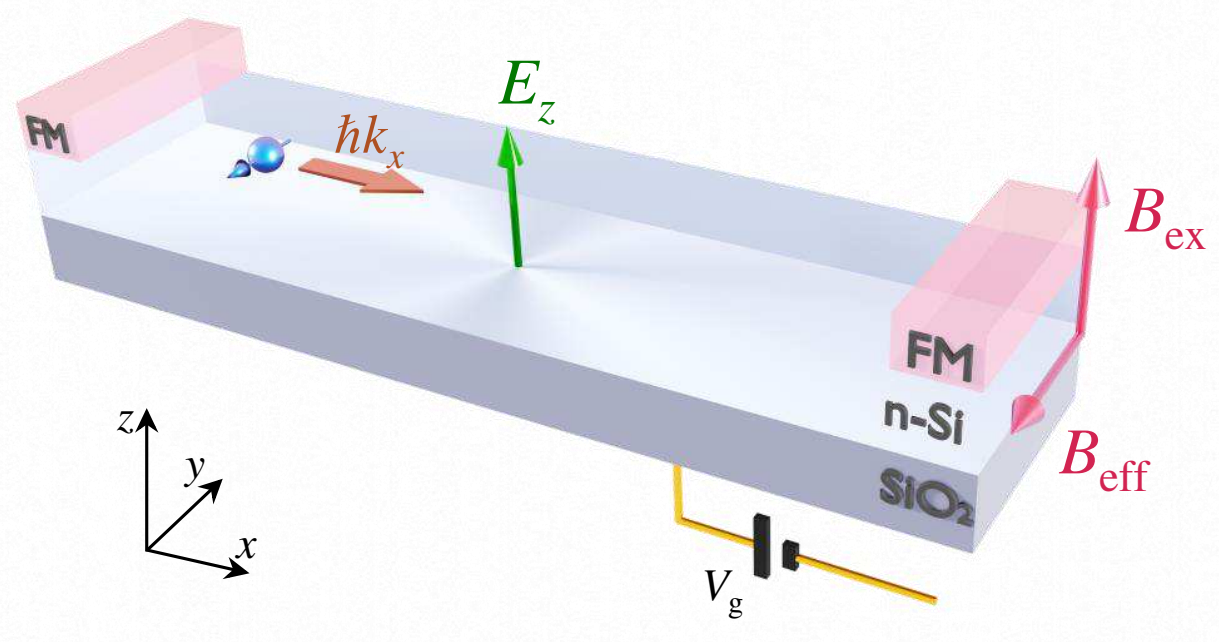

(b)

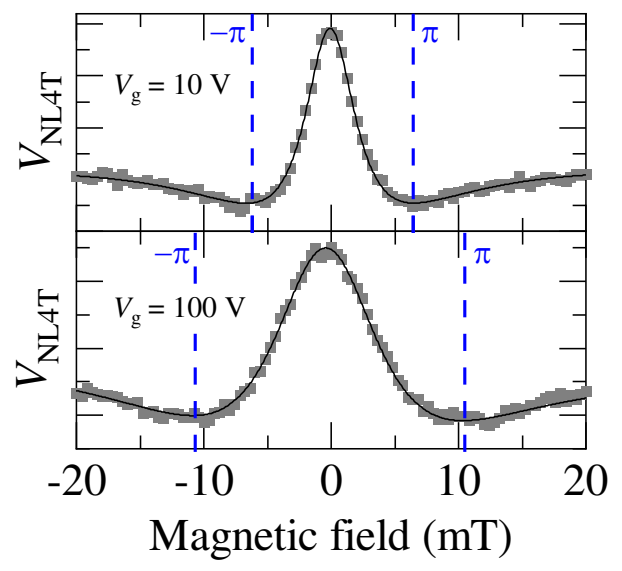

(c)

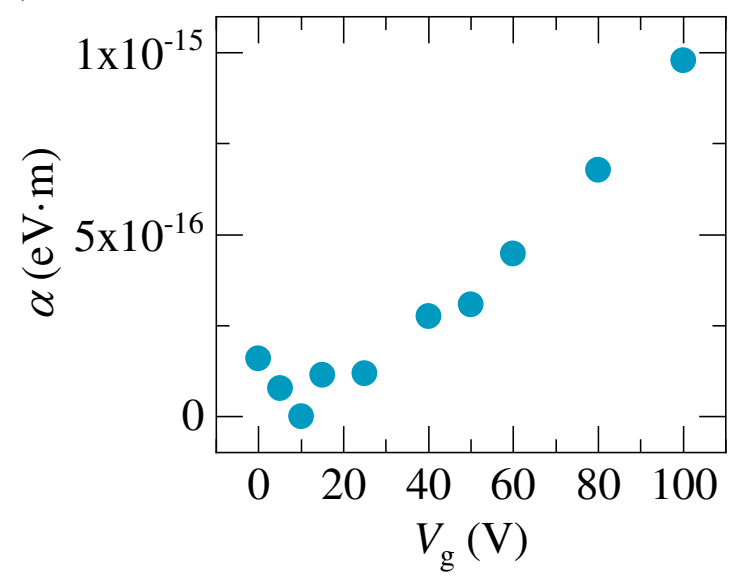

Figure 4. | Physics behind the spin lifetime anisotropy, the Rashba SOI and the emergent effective magnetic field in the $\mathrm{Si}$ MOS, and the gate-tuneable Rashba parameter. (a) Schematic illustration of the synthetic Rashba SOI, resulting in the emergent effective magnetic field in the Si MOS. A gate electric field is applied perpendicular to the Si MOS plane $\left(E_{z}\right)$, and the spin momentum is parallel to the plane $\left(\hbar k_{x}\right)$. Consequently, an emergent effective magnetic field, $B_{\text {eff, }}$ is generated along the $-y$-direction due to the Rashba SOI. An external magnetic field is applied perpendicular to the plane $\left(B_{\mathrm{ex}}\right)$, which allows spin precession of the in-plane spins. (b) Spin precession signals at $\beta=90^{\circ}$, where $V_{\mathrm{G}}$ is set to $10 \mathrm{~V}$ and $100 \mathrm{~V}$. Grey dots are experimental 
data, and black solid lines are the fitting results. Blue dashed lines indicate the magnetic fields for averaged $\pi$ - and $-\pi$-rotation of spins accumulated below the detector. (c) $V_{\mathrm{g}}$ dependence of the Rashba parameter $(\alpha)$. Given that $\zeta$ is almost 1 (isotropic spin lifetime) at $V_{\mathrm{g}}=10 \mathrm{~V}$, we postulate that the Rashba SOI at $V_{\mathrm{g}}=10 \mathrm{~V}$ is negligible in the estimation of $\alpha$. 
(a)

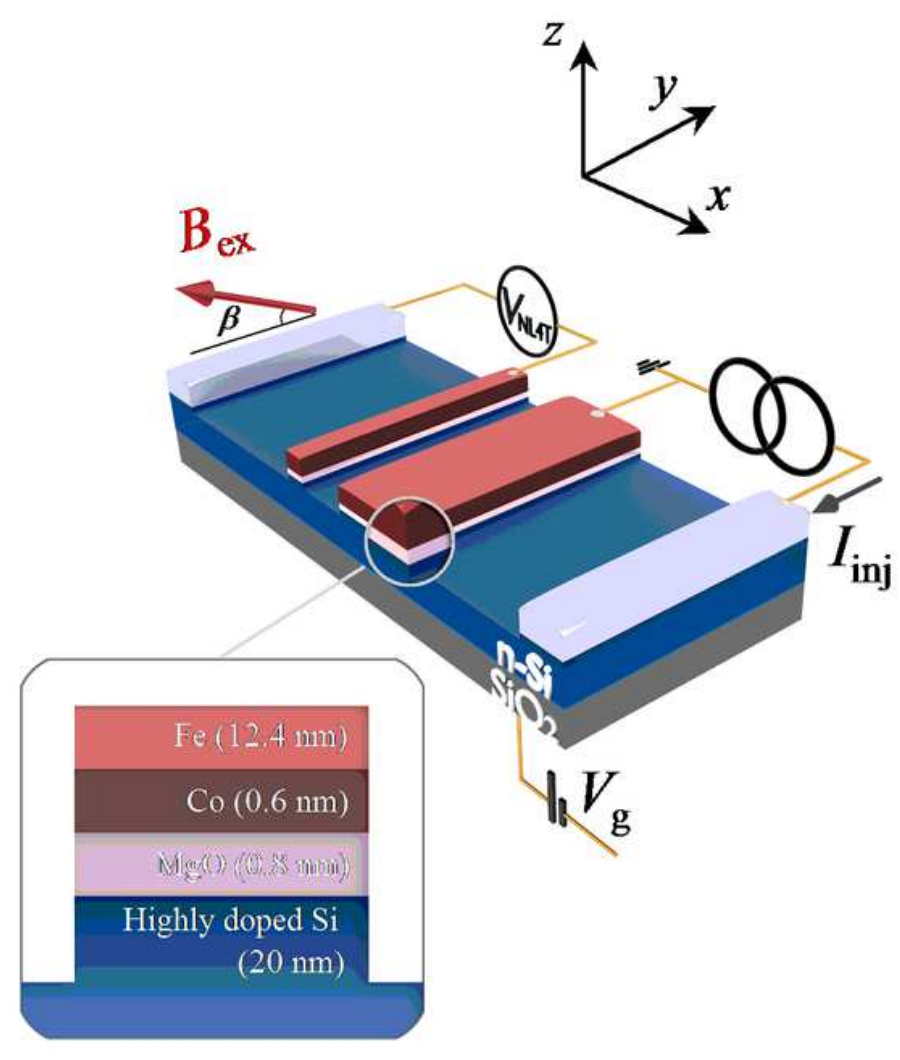

(b)

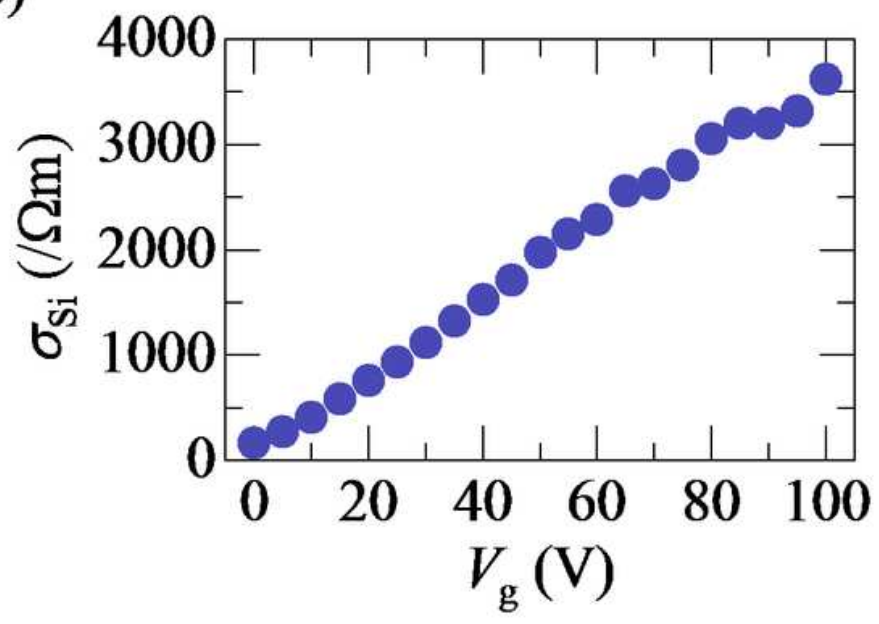

(c)

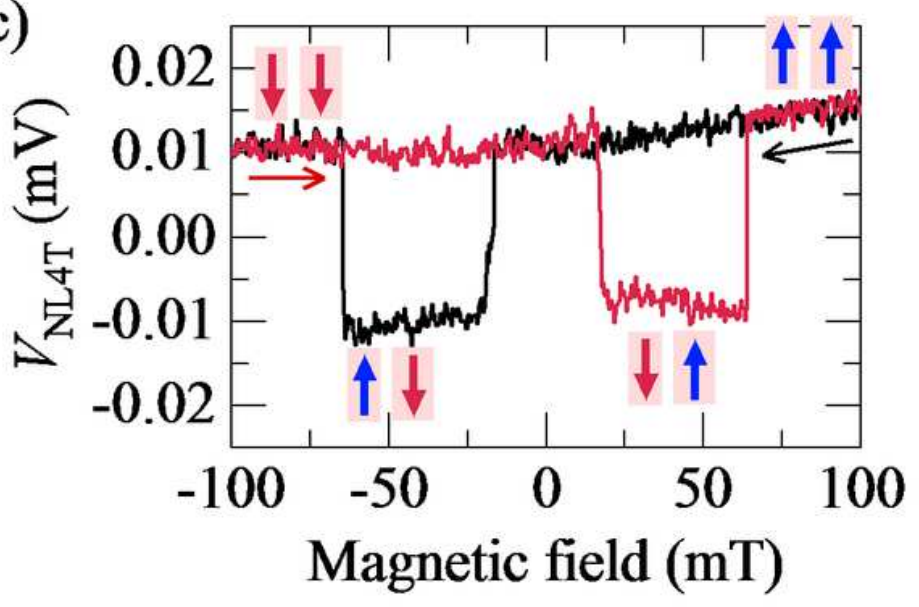

Figure 1

Device structure and basic charge and spin transport characteristics in a synthetic Rashba system made of Si MOS. (a) Schematic image of a synthetic Rashba device made of Si MOS and the measurement setup. The Si MOS channel is non-degenerate and $100 \mathrm{~nm}$ thick. An electric current (linj) is applied between one ferromagnetic contact and one non-magnetic contact, and NL4T voltages (VNL4T) are measured in the separated circuit including a ferromagnetic contact and a non-magnetic contact: nonlocal alignment. An external magnetic field (Bex) is applied in the y-z plane with a tilt angle of $\beta$. (b) Conductivity of the Si MOS ( $\sigma \mathrm{Si}$ ) as a function of gate voltage $(\mathrm{Vg})$. $\sigma S i$ was measured using a conventional four-terminal method. (c) Typical non-local magnetoresistance ( $\triangle \mathrm{VNL} 4 \mathrm{~T})$ as a manifestation of successful spin propagation in the Si MOS. An in-plane $\left(\beta=0^{\circ}\right)$ external magnetic field was swept upwards (red solid line) and downwards (black solid line). 
(a)

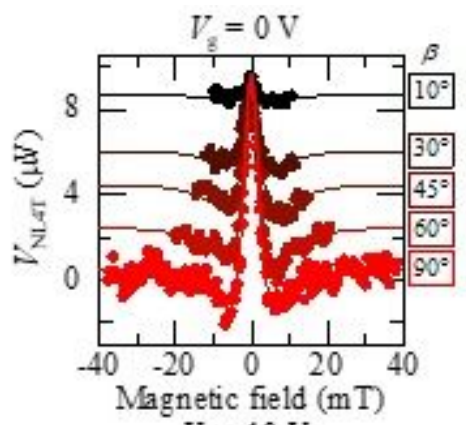

(b)

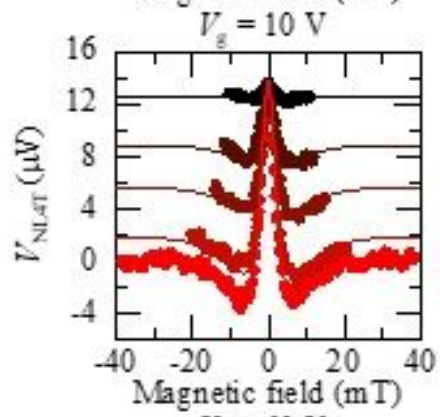

(c)

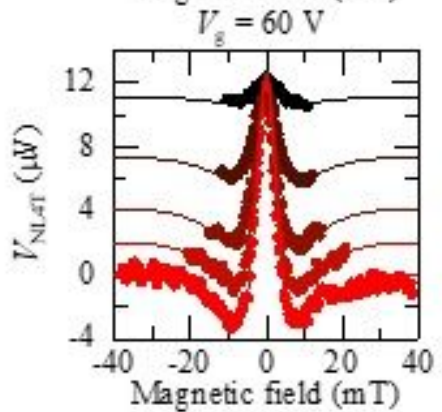

(d)

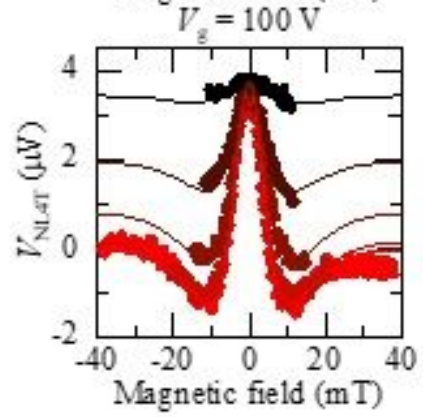

(e)

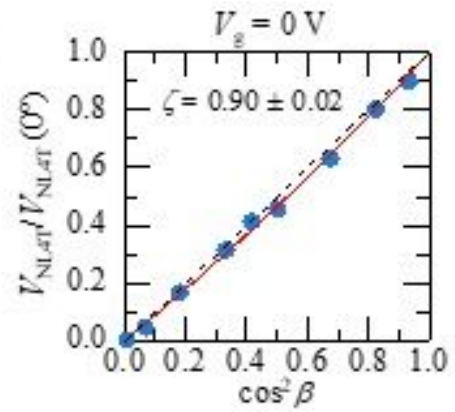

(f)

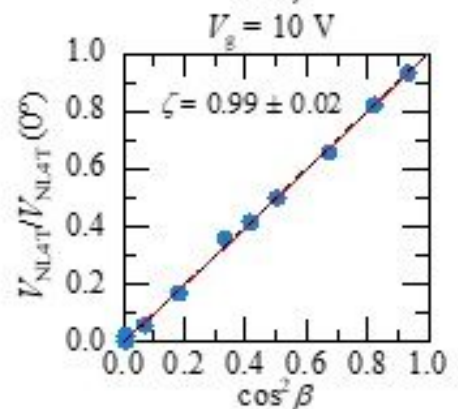

(g)

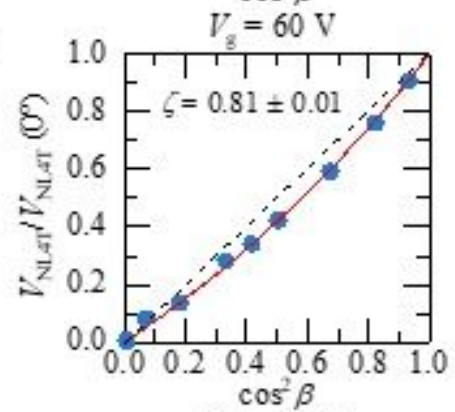

(h)

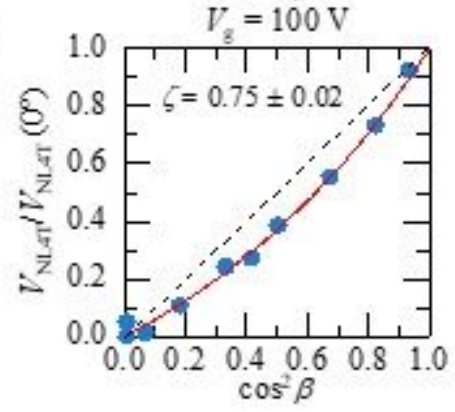

\section{Figure 2}

Gate modulation of spin precession signals and spin lifetime anisotropy. (a)-(d) Non-local spin signal as a function of tilt angle $\beta$ and gate voltage $\mathrm{Vg}$. Solid lines show fitting results obtained using a conventional spin precession equation. (e)-(h) Spin signals under a high magnetic field (>40 mT) at various $\beta$ normalized by those at $\beta=0^{\circ}$ as a function of $\cos 2 \beta$. Red solid lines are fitting results obtained by using Eq. (1) in the main text. The anisotropy ratio of the spin lifetime ( $\zeta)$ is extracted by fitting and is depicted in the figures. The black dashed lines represent the $\cos 2 \beta$ dependence when $\zeta=1$ (isotropic). 


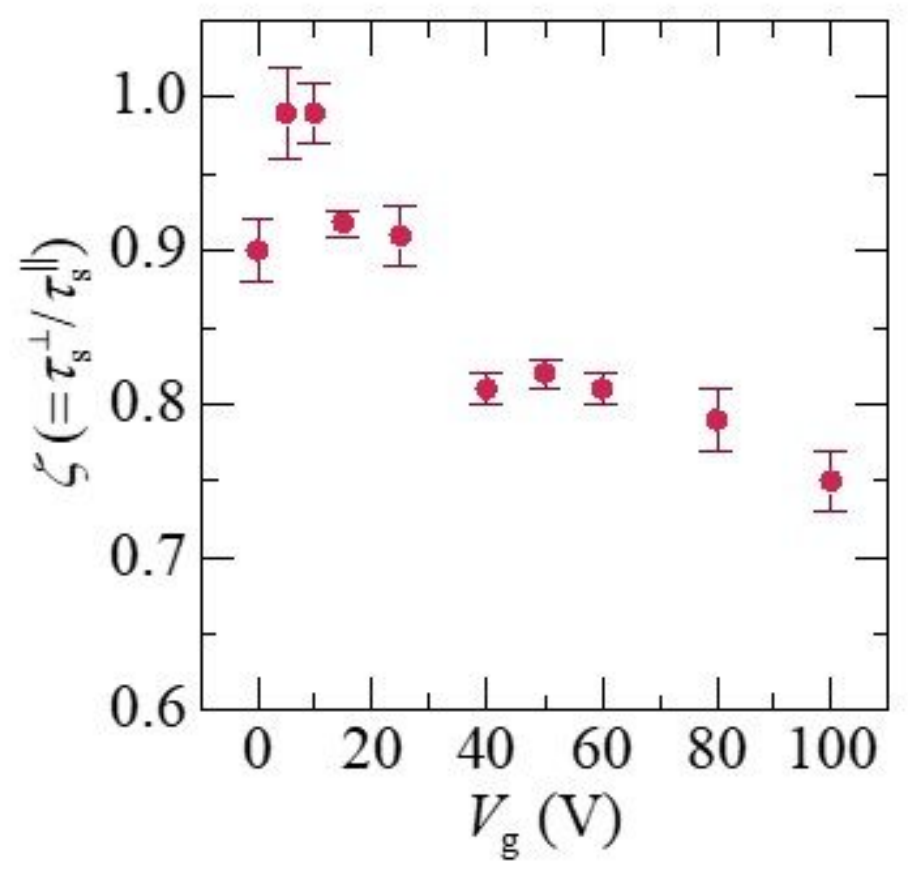

\section{Figure 3}

Gate voltage dependence of the spin lifetime anisotropy. The spin lifetime anisotropy ratio $\zeta$ is dependent on $\mathrm{Vg}$, and it is the largest and almost unity at $\mathrm{Vg}=10 \mathrm{~V}$, implying an isotropic spin lifetime. Above $\mathrm{Vg}=$ $10 \mathrm{~V}, \zeta$ monotonically decreases with increasing $\mathrm{Vg}$, which is a manifestation of the generation and enhancement of a synthetic Rashba SOI field, resulting in an emergent effective magnetic field. The error bars are estimated as the standard deviation in the fitting. 
(a)

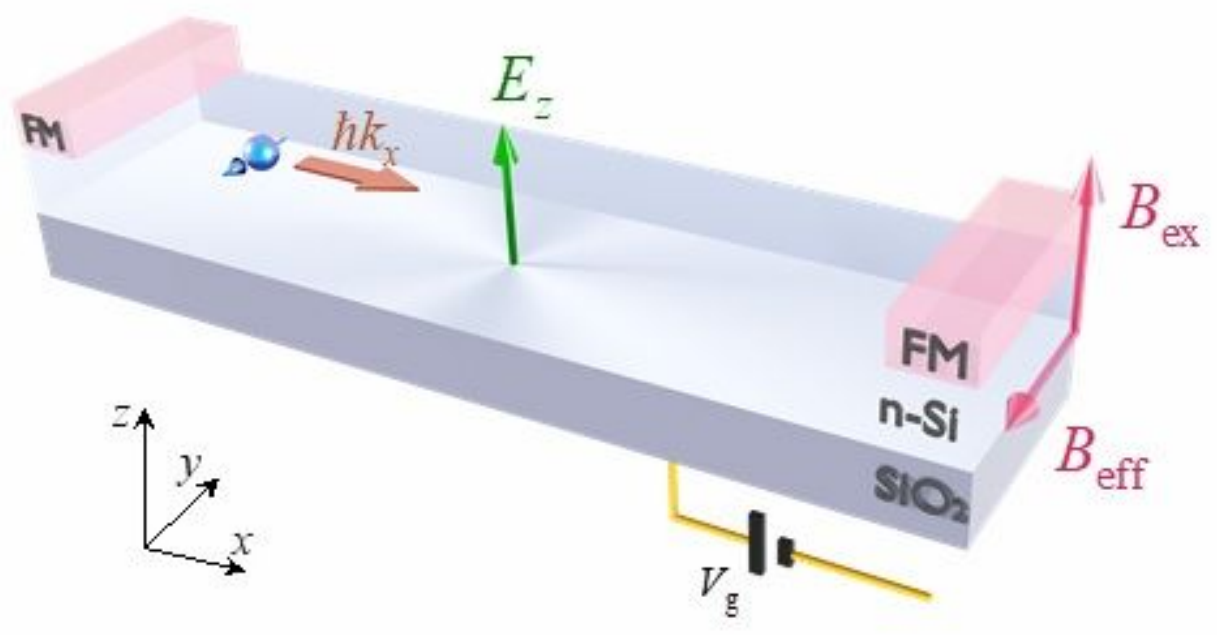

(b)

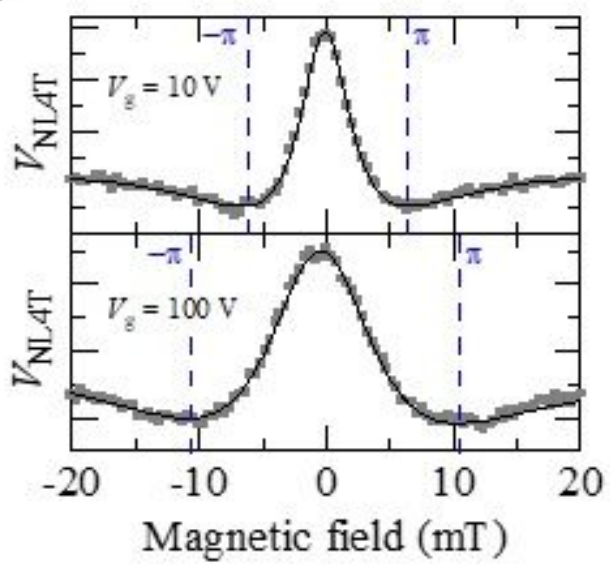

(c)

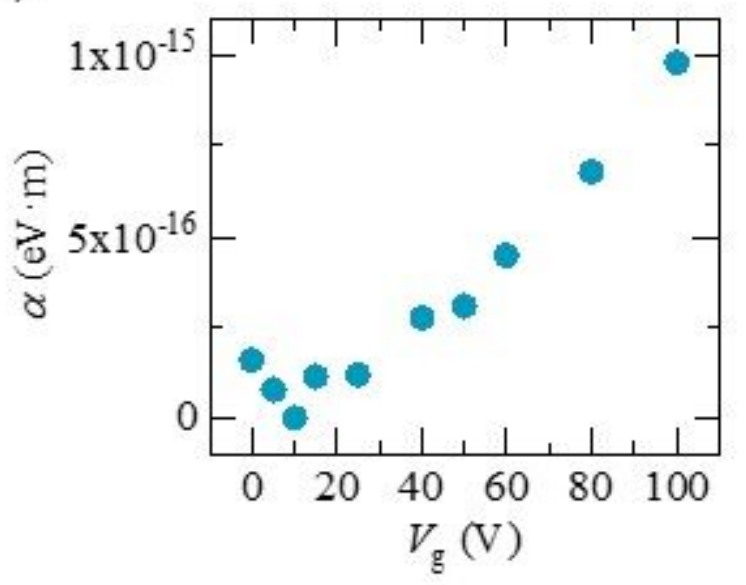

Figure 4

Physics behind the spin lifetime anisotropy, the Rashba SOI and the emergent effective magnetic field in the Si MOS, and the gate-tuneable Rashba parameter. (a) Schematic illustration of the synthetic Rashba SOI, resulting in the emergent effective magnetic field in the Si MOS. A gate electric field is applied perpendicular to the Si MOS plane (Ez), and the spin momentum is parallel to the plane (" $\| " \mathrm{kx}$ ). Consequently, an emergent effective magnetic field, Beff, is generated along the -y-direction due to the Rashba SOI. An external magnetic field is applied perpendicular to the plane (Bex), which allows spin precession of the in-plane spins. (b) Spin precession signals at $\beta=90^{\circ}$, where VG is set to $10 \mathrm{~V}$ and $100 \mathrm{~V}$. Grey dots are experimental data, and black solid lines are the fitting results. Blue dashed lines indicate the magnetic fields for averaged $\pi$ - and - $囚$-rotation of spins accumulated below the detector. (c) $\mathrm{Vg}$ dependence of the Rashba parameter (a). Given that $\zeta$ is almost 1 (isotropic spin lifetime) at $\mathrm{Vg}=10 \mathrm{~V}$, we postulate that the Rashba SOI at $\mathrm{Vg}=10 \mathrm{~V}$ is negligible in the estimation of $\mathrm{a}$. 


\section{Supplementary Files}

This is a list of supplementary files associated with this preprint. Click to download.

- LeeNMSIsubmission.docx 